\title{
Hypoxia-Inducible Factor-2a Promotes EMT in Esophageal Squamous Cell Carcinoma Through Notch Pathway
}

\section{Zhiqi Ji}

Shandong University School of Medicine: Shandong University Cheeloo College of Medicine

Xiangyan Liu (D liuxy6638@126.com)

Shandong Provincial Hospital https://orcid.org/0000-0002-6998-1543

\section{Shihao Bao}

Shandong University Cheeloo College of Medicine

\section{Liang Li}

Shandong University Cheeloo College of Medicine

Mo Shi

Shandong Provincial Hospital

Liang Song

Shandong Provincial Hospital

Dong Wang

Shandong Provincial Hospital

Yang Yu

Shandong Provincial Hospital

\section{Research Article}

Keywords: hif-2a, Notch, EMT, ESCC

Posted Date: June 3rd, 2021

DOl: https://doi.org/10.21203/rs.3.rs-535539/v1

License: (c) (1) This work is licensed under a Creative Commons Attribution 4.0 International License. Read Full License

Version of Record: A version of this preprint was published at Advances in Clinical and Experimental Medicine on April 8th, 2022. See the published version at https://doi.org/10.17219/acem/147270. 


\section{Abstract}

Esophageal squamous cell carcinoma (ESCC) is one of the most common devasting tumor around the world, characterized by persistent hypoxia. Hypoxia-inducible factor-2a (hif-2a) has been regarded as an oncogene in many kinds of cancers, exerting its function to promote tumor growth and metastasis. But the role of hif-2a in ESCC remains largely unknown. In this study, we discovered hif-2a was overexpressed in ESCC and could promote proliferation and metastasis in ESCC cells. Functionally, hif-2a facilitated EMT through Notch pathway in ESCC. Moreover, we identified hif-2a as an independent factor that predict poor prognosis in ESCC patients. These findings suggested that hif-2a is a key regulator of EMT and played a critical role in promoting ESCC progression.

\section{Introduction}

Esophageal cancer is one of the most lethal tumor around the world, and the most common histology kind in China is esophageal squamous cell carcinoma (ESCC), accounting for $90 \%[1]$. Esophageal cancer occurs at a high incidence in certain areas, among which China is the area with highest incidence[1]. Although various of therapy strategies has been used in clinical treatment, the 5-year survival rate is still not satisfied, only $15 \%-20 \%[2]$. The reason for poor prognosis of ESCC is that the tumor is easy to occur distant metastasis. However, the mechanism of metastasis has not been studied clearly.

It has been reported that hypoxia can induce ESCC undergo EMT process through several mechanisms, in which hypoxia-inducible factor $1 \mathrm{a}(\mathrm{hif}-1 \mathrm{a})$ plays an important role[3]. Overexpression of hif-1a correlates with lymph node metastasis and poor prognosis in patient with ESCC[4]. However, studies suggested that hif- $1 a$ is usually induced in severe hypoxia conditions to make cells resistant to hypoxia. Hif-2a, a member of hypoxia inducible factor family, homologous to hif- $1 a$, has been reported to play important roles in several cancers. It has been reported that hif-2a can regulate EMT process through the crosstalk with $\mathrm{Wnt} / \beta$-catenin signaling in pancreatic cancer[5]. However, there has no studies of hif-2a in ESCC yet, the role of hif-2a in ESCC is still unknown, thus need further investigation.

The Notch signaling pathway is an evolutionary conserved local cell signaling pathway that participates in a variety of cellular processes: cell differentiation, proliferation, apoptosis, adhesion, epithelialmesenchymal transition, migration, and angiogenesis, affecting embryonic development, tissue balance, and the body immunity[6]. It has been reported to act as an oncogene in various cancers, including lung cancer, breast cancer, colorectal cancer, prostate cancer, T-ALL and other malignancies[7]. Notch signaling has been proved to have crosstalk with HIF-1a in some types of cancers which consequently result in changes of EMT markers, such as breast cancer[8]. Moreover, some researches have shown that aberrant activation of notch signaling plays important roles in progression of ESCC and predict poor prognosis in ESCC patients[9]. However, the intensive mechanism of notch signaling in ESCC remains unknown, especially how notch regulate EMT process, thus need further investigation.

\section{Result}




\section{Hif-2a is overexpressed in ESCC}

To investigate whether hif-2a is overexpressed in ESCC tissues and cell lines, Immunohistochemistry and immunofluorescence were performed. Immunohistochemistry shows that ESCC tissues express high level of hif-2a while hif-2a can be hardly detected in normal esophageal epithelial (Figure 1a). Besides, the result shows that hif-2a is mainly aggregated in nucleus, this is consistent with the transcription factor role of hif-2a. Immunofluorescence suggested that hif-2a can be detected in ESCC cell lines Eca109 and KYSE150 under normoxia conditions, located mainly in nucleus, consistent with the Immunohistochemistry result (Figure 1b).

\section{Hif-2a is associated with hypoxia-induced EMT in ESCC}

Two esophageal cancer cell lines were used to mimic hypoxia induced EMT.As expected, morphological changes of the cells were observed after treated with hypoxia condition for 48h (Figure 2a), accompanied by decreased expression of E-cadherin, increased expression of $\mathrm{N}$-cadherin, vimentin and snail (Figure $2 b)$. We also used Cocl2 to mimic hypoxia, and decreased expression of E-cadherin, increased expression of $\mathrm{N}$-cadherin, vimentin and snail were also observed (Figure 2c).

\section{Hif-2a promote proliferation and invasion of esophageal cells}

Hif-2a has been reported can promote cell proliferation and invasion in different tumors, but the role of Hif-2a in esophageal cancer cell has not been studied yet. To investigate whether Hif-2a could promote proliferation and invasion in esophageal cells, CCK8 and transwell assay were performed. CCK8 assay shows the OD value(450nm) of hif-2a silencing group is significantly decreasing at $24 \mathrm{~h}, 48 \mathrm{~h}, 72 \mathrm{~h}, 96 \mathrm{~h}$ both in Eca-109 and KYSE-150 ESCC cells (Figure 3a). Moreover, silencing hif-2a showed a stronger inhibitory effect on proliferation in Eca-109 cells. As for transwell assay, compared to the control and NCsiRNA groups, The number of hif-2a silencing ESCC cells entering the Transwell membrane was significantly reduced, suggesting the invasion capability of ESCC cells was suppressed in case of hif-2a knockout (Figure $3 b, d$ ). In contrast, the hif-2a overexpressed group showed a significantly increased cell numbers penetrating the membrane compared to control and NC-OE group, indicating the overexpression of hif-2a enhanced the invasion ability of ESCC cells(Figure $3 c, e)$. These results suggest that hif-2a plays an important role in proliferation and invasion in ESCC cells.

\section{Hif-2a regulate EMT through Notch pathway}

To determine if hif-2a could regulate EMT in ESCC cells, western blot assay was performed to detect the protein levels of EMT markers. Compared to the control and NC-siRNA groups, silencing group showed increased level in E-cadherin and decreased expression level in $\mathrm{N}$-cadherin, Vimentin and snail both in Eca-109 and KYSE-150 ESCC cells (Figure 4a), while the result of overexpressed group is contrast (Figure 4b). Besides, the expression level of NICD, which is the active form of Notch and functions as transcriptional regulators, shows a same trend with hif-2a (Figure $4 a, b)$. These results showed both EMT and Notch could be regulated by hif-2a. Then the inhibitor CB-103 was used to study if Notch pathway 
could regulate EMT, which is a specific and high effect inhibitor of Notch pathway. The result showed CB103 could significantly reverse the EMT process in ESCC cells (Figure 4c). Furthermore, retrial experiment was carried out to investigate if it was through Notch pathway that hif-2a regulated EMT in ESCC cells. It could be observed that EMT process was reversed in hif-2a overexpressed ESCC cells after the Notch pathway inhibitor was used (Figure $4 d$ ). These results suggest that hif-2a could regulate EMT through Notch pathway in ESCC cells.

\section{Silencing hif-2a could suppress the tumor growth in mouse model.}

Xenograft mouse model was used to confirm the effect of hif-2a in vivo. Compared to the NC group, the tumor volume and weight of siRNA were obviously decreased (Figure 5a-d), while no significant difference was observed in the weight of nude mice (Figure 5e). This result suggested that inhibiting hif$2 a$ significantly suppress the growth of tumor without any apparent impacts on mouse growth. Immunohistochemistry and western blot were performed using the xenograft tumor to confirm that hif-2a could regulate EMT through NOTCH pathway, the results showed hif-2a knockout could significantly suppressed Notch signaling and reverse EMT in vivo (Figure $5 \mathrm{f}, \mathrm{g}$ ).

\section{Overexpression of hif-2a is associated with poor prognosis in ESCC patients.}

We studied the relationship between hif-2a expression level and clinical factors in ESCC patients. The expression level of hif-2a was divided into high and low groups according to the $\mathrm{H}$-score. The overexpression of hif-2a was significantly associated with lymph node metastasis $(p=0.005)$ and tumor stage $(p=0.016)$ (Table 1). Interesting, a correlation between gender and hif-2a overexpression was found $(p=0.038)$ (Table 1). Besides, hif-2a expression level could be an independent factor for prognosis with a hazard ratio of $4.538(95 \% \mathrm{Cl}=1.426-14.44, \mathrm{p}=0.01)$ (Table 2$)$. Moreover, lymph node metastasis could also be a prognosis factor with a hazard ratio of $2.144(95 \% \mathrm{Cl}=1.284-4.527, \mathrm{p}=0.006)$ (Table 2). To investigate the relationship between the hif-2a expression level and OS of patients with ESCC, a kaplanmeier curve was made and the result showed patients with low expression level of hif-2a would had much better overall survival $(p=0.015)$ (Figure 6).

Table 1 Association between hif-2a expression and clinicopathological information in 100 ESCC patients 


\begin{tabular}{|c|c|c|c|}
\hline & hif-2a low & hif-2a high & $P$ value \\
\hline gender & & & \\
\hline male & 38 & 50 & 0.038 \\
\hline female & 9 & 3 & \\
\hline age & & & 0.153 \\
\hline$\llbracket 60$ & 28 & 24 & \\
\hline$\geq 60$ & 19 & 29 & \\
\hline Maximal dimension of tumor & & & 0.897 \\
\hline$\varangle 5$ & 26 & 30 & \\
\hline$\geq 5$ & 21 & 23 & \\
\hline Lymph node metastasis & & & 0.005 \\
\hline yes & 17 & 34 & \\
\hline no & 30 & 19 & \\
\hline Stage & & & 0.016 \\
\hline I/II & 30 & 21 & \\
\hline III/IV & 17 & 32 & \\
\hline
\end{tabular}

Table 2 Cox regression analysis of indicated factors for patient survival

\begin{tabular}{|c|c|c|}
\hline & $\mathrm{HR}(95 \% \mathrm{Cl})$ & $P$ value \\
\hline Age $(\geq 60 / \otimes 60)$ & $1.008 \rrbracket 0.573-1.775 \rrbracket$ & 0.978 \\
\hline Gender $\$ male/female $\rrbracket$ & $1.204 \rrbracket 0.542-2.676 \rrbracket$ & 0.648 \\
\hline Hif-2a囚high/low $\square$ & $4.538 \rrbracket 1.426-14.44 \rrbracket$ & 0.01 \\
\hline 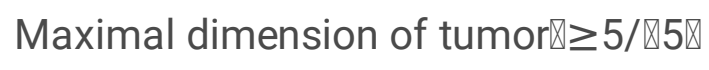 & $1.174 \rrbracket 0.265-5.204 \rrbracket$ & 0.833 \\
\hline Lymph node metastasis『yes/no》 & 2.411ه1.284-4.527区 & 0.006 \\
\hline 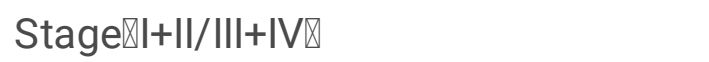 & $0.207 \otimes 0.37-1.155 \rrbracket$ & 0.073 \\
\hline
\end{tabular}

\section{Discussion}


Esophageal squamous cell carcinoma is one of the most common malignant tumors of the digestive tract, characterized by poor quality of life and low five-year survival rate. Although the treatment plan has improved significantly, surgical resection is still considered the first line of treatment for early and local ESCC. However, ESCC is generally resistant to conventional therapeutic agents. In addition, the latest ESCC inhibitors are clinically ineffective. Therefore, finding new biological targets that may be useful to improve the prognosis of patients with ESCC has always been a central issue in recent years.

Hypoxia inducible factor (HIF) has been researched widely and deeply in the past decades. It is a key regulator in cells responsible to prevent damage from hypoxia, which was mainly regulated by the oxygen level in cells[10]. Under the normoxia conditions, HIF was modified by HIF-specific prolyl-hydroxylases (PHDs), which lead to its degradation by Von Hippel Lindau tumor suppressor (pVHL)[11]. When the cell is in hypoxia condition, PHDs was inactivation and the VHL-dependent degradation pathway was blocked, causing the accumulation of HIF and activation of downstream genes. As a member of HIF, hif$2 a$ has been reported to associate with poor prognosis in various cancers, such as NSCLC, breast cancer, colorectal cancer, hepatocellular cancer and glioblastoma etc[12]. But the role of hif-2a in ESCC has not been studied yet. In this study, we identified hif-2a as a key molecule to regulate EMT and it can be an independent factor of prognosis in ESCC patients. We found that hif-2a was overexpressed both in ESCC tissues and cell lines. The overexpression of hif-2a in tumor tissue might be due to the hypoxia tumor microenvironment, but the mechanism of why hif-2a is overexpressed under normoxia in ESCC cell lines remain unclear. Th possible hypothesis could be increased transcription or impacted degradation pathway of hif-2a, which needs further investigation. Besides, we discovered the function of hif-2a to promote proliferation and metastasis in ESCC. Both the in vitro and in vivo experiments verified the role of hif-2a in promoting growth of ESCC cells. And the function of hif-2a to promote metastasis and regulate expression of EMT markers was also confirmed in our investigation. In addition, we confirmed that hif-2a associated with poor prognosis in patients with ESCC, characterized by lower five-year survival rate and higher incidence of lymph node metastasis.

Notch signaling pathway has been studied for over one hundred years since it was first described by Thomas Hunt Morgan in 1917. Previous studies have proved that Notch functions as an oncogene in many kinds of cancers, and the mechanism of how Notch signaling pathway works has been interpreted clearly. The transduction process of Notch signaling pathway mainly includes the classical pathway that relies on CSL and CSL-independent transduction pathways. The classical pathway of Notch signaling is triggered by the interaction between the Notch ligand and the receptor, then the Notch receptor releases the active form of the Notch protein, that is, the intracellular segment of ICN (NICD)[13]. The NICD enters the nucleus, where it acts as a transcription regulator. In the past decades of years, it has been confirmed that Notch signaling played important roles in regulating EMT process in different cancers, including esophageal cancer[14]. The crosstalk between signaling pathways in cell is an extremely complicated network, not except for Notch signaling. It has been reported that both hif- $1 a$ and hif-2a could regulate Notch signaling in different cancers respectively[15, 16]. But the relationship between HIF signaling and Notch signaling in ESCC remains unclear. In this study, Notch signaling was found to be a downstream of hif-2a. We observed the expression of NICD, the active form of NOTCH, could be regulated by hif-2a. As a 
modulator of EMT, NICD could enter the nucleus and regulate transcription of downstream genes. Both the EMT markers and NICD could be regulated by hif-2a, but whether EMT was regulated by hif-2a through Notch signaling pathway remains unknown. So we carried out the retrieval experiment and the result verified our speculation that Notch was the intermediary of hif-2a to regulate EMT in ESCC. But the mechanism of how hif-2a regulate Notch signaling was not clear and need further investigation.

\section{Materials And Methods}

\section{Patients}

100 patients who were diagnosed with ESCC and underwent esophageal cancer operation in the Department of Thoracic Surgery at Shandong Provincial Hospital from January 2014 to December 2015 were included in this project. The inclusion and exclusion criteria were as follows:

1. esophageal squamous cell carcinoma was confirmed by pathology after the surgery;

2. chemotherapy and radiotherapy were not used on patients before surgery;

3. contraindications of surgery were excluded by preoperative examination;

4. patients who died by complications or accident after surgery were excluded;

The tumor was excised completely in all patients: No tumors visible to the naked eye in the surgical field; the upper and lower resection margin were cancer cell negative by pathology; the TNM stage was determined by International Union Against Cancer in 2009 in 2017. This project was supported by the institutional ethics committee of Shandong Provincial Hospital Affiliated to Shandong University according to Guide for Chinese Ethics Review Committee and all the patients were agreed with the Written informed consent.

\section{Cell culture and reagents}

Esophageal squamous cell carcinoma (ESCC) cell lines (Eca109 and KYSE150) were obtained from the Shanghai Institute for Biological Science (Shanghai, China). KYSE-150 was cultured in Roswell Park Memorial Institute (RPMI)-1640 Medium (GE Healthcare Life Science, Logan, UT, USA). Eca-109 was cultured in high glucose Dulbecco's modified Eagle's medium (DMEM; Gibco, Logan, UT, USA). Both media were supplemented with $10 \%$ fetal bovine serum(FBS,BI). All cells were cultured at $37.0 \pm 0.2^{\circ} \mathrm{C}$ in a humidified incubator with $5.0 \% \mathrm{CO} 2$. Before exposure to the hypoxic environment $(3.0 \% \mathrm{O} 2)$, cells were cultured in normoxia conditions and grown to about $60 \%$ confluence. Then cells were cultured in a lowoxygen environment in a hypoxic incubator(Thermal Tech, Orlando, FL, USA) for the indicated duration.

Cobalt chloride (CoCl2) was purchased from Sigma-Aldrich (St. Louis, MO, USA), and was used at $50 \mathrm{mM}$ for $48 \mathrm{~h}$. Notch antagonist CB-103 was purchased from MCE(HY-135145), and was used at 20mM for 48 h.

\section{Transfection}


The hif-2a overexpression and silencing lentivirus were designed and purchased from GeneChem Company(Shanghai, China). The virus was transfected into cells according to the protocol of Genechem. After the virus were added into cells, puromycin (58-58-2, Solarbio) was used at the concentration of 5 $\mathrm{ug} / \mathrm{ml}$ to screen the stable transposable.

\section{CCK-8 assay}

Prior to using Cell Counting Kit 8 (CCK-8), cells were seeded in 96-well plates with 2000 cells per well for specific treatments. Cell viability was evaluated using CCK-8 (Dojindo, Kumamoto, Japan). After incubating the cells with CCK-8 working solution for 30 minutes, absorbance was measured at $450 \mathrm{~nm}$ using an ELx808 microplate reader (BioTeck). Relative cell viability was expressed as a percentage of a particular control.

\section{Transwell assay}

The tumor cell infiltration activity was evaluated by its ability to penetrate the gel matrix (Matrigel; BD, Franklin Lakes, NJ, USA). In short, the Matrigel solution was diluted 1:8 in FBS-free medium and filtered with $6.5 \mathrm{~mm}$ diameter (8 micron holes) polycarbonate in a 24-well Transwell box (Corning, NY, USA) Film coverage. All filters are filled with $40 \mathrm{ml}$ of working solution, and the plates are cured at $37^{\circ} \mathrm{C}$ for at least 5 hours. Seed tumor cells at a density of $2 \times 105$ in each chamber, and culture in the upper compartment of the chamber in FBS-free medium for 48 hours, and the lower chamber is filled with complete medium. Wipe the filter with a cotton swab, and fix the cells attached to the bottom with $4 \%$ formaldehyde polyphosphate (Beyotime, Shanghai, China). After staining with crystal violet (Beyotime), these cells with greater infiltration capacity were counted. The experiment was repeated three times independently.

\section{Immunohistochemistry and immunofluorescence}

The hif-2a immunohistochemistry study was carried out by the streptavidin peroxidase method. The formalin fixed and paraffin embedded ESCC tissue was cut into 5- $\mu \mathrm{m}$ thick sections and then being deparaffinized and incubated with hydrogen peroxide. The rabbit anti-hif-2a antibody (NB100-122, Novus, USA) was diluted 1: 200 and incubated overnight at $4{ }^{\circ} \mathrm{C}$. The next step was followed by the instruction of secondary biotinylated antibody kit (Zhongshan Biotech, Guangzhou, People's Republic of China). The stained slides were evaluated by 2 observers independently.

Before the immunofluorescence staining was performed, $4 \%$ polyphosphate formaldehyde was used to fix the cells for 15 minutes. After being penetrated by $1 \%$ Triton for 30 minutes, the cells were blocked with goat serum for 1 hour at room temperature. Then the cells were incubated over night at $4{ }^{\circ} \mathrm{C}$, with rabbit anti-hif-2a antibody (NB100-122, Novus), and were incubated with the secondary antibodies conjugated to fluorescent dyes for 1 hour at room temperature in the dark. After counterstained with 4'6diamidino-2-phenylindole (DAPI)(C0065, Solarbio)for 5minutes, the cells were observed under an inversion fluorescence microscope.

\section{Protein extraction and western blotting}


The protein was extracted using the Ripa lysis reagent (R0010, Solarbio). The protein sample was electrophoresed on an SDS polyacrylamide gel and transferred to a PVDF filter membrane (Millipore Corporation, Billerica, Mass., USA). Membranes were incubated overnight with primary antibodies after blocked with non-fat milk. Primary antibodies were used as follows: (anti-E-cadherin, anti-N-cadherin, antiVimentin and anti-snail were obtained from Cell Signaling Technology; anti- $\beta$-actin was purchased from Boster (Biological Technology Co. Ltd., CA, USA). After washing, the membrane was incubated with a secondary antibody bound to anti-rabbit IgG horseradish peroxidase (Boster). The band was visualized by an improved chemiluminescence detection system (Amersham imager 680; General Electric, Fairfield, CT, USA).

\section{Mouse model}

Eca-109 cell transfected with siRNA and negative-control lentivirus were used to investigate the effect of hif-2a on tumor progression in vitro. $1 \times 106 \mathrm{NC}$ and hif-2a silencing cells were independently injected in the flank subcutaneously of five-week-old female BALB/c nude mice. Then the tumor sizes were measured every five days and weighed after 20 days. The formula $V=L \times W 2 / 2$ was used to calculated the volume of tumor, in which $L$ is the largest and $w$ is the smallest diameter. The protocol was approved by the ethics committee of Provincial Hospital affiliated to Shandong First Medical University.

\section{Statistical Analysis}

The statistical data were presented in the form of mean \pm standard. $P$ values were calculated by the analysis of variance and Chi-squared test. It is considered to be statistically different if $P$ value $<0.05$. All statistical calculations and graph were performed using SPSS (version 24.0) and GraphPad Prism 7 statistical software.

\section{Conclusion}

In general, this project illuminated a pro-tumor tole of hif-2a in esophageal cancer. Hif-2a enhanced proliferation and invasion ability of ESCC cells, promoted EMT through Notch signaling pathway. Besides, we recognized hif-2a as an independent indicator of poor prognosis in ESCC patients, associated with shorter OS and lymph node metastasis. Hence, hif-2a may become a potential target for treating esophageal cancer and we might have new strategy to prolong the survival time of ESCC patients.

\section{Declarations}

\section{Acknowledgement}

The authors are very grateful to the help from the Department of Thoracic Surgery, Pathology Department and Central Laboratory of Shandong Provincial Hospital affiliated to Shandong First Medical University.

\section{Funding}


This work was financially supported by the Natural Science Foundation of Shandong Province (ZR2020MH244), National Natural Science Foundation of China (81902418), Natural Science Foundation of Shandong Province (ZR2019MH092).

\section{Author contributions}

ZQ $\mathrm{J}$ conceived and designed the study. SH B and LL performed the experiments. LS and YY analyzed the data. MS and DW wrote the manuscript. All the authors read and approved the final paper.

\section{Data availability}

All raw data are available on case of reasonable request from corresponding author.

\section{Compliance with ethical standards}

Conflict of interest: The authors declare that they have no conflict of interest.

Ethical approval and consent to participate: The study was approved by ethics committee of institutional ethics committee of Shandong Provincial Hospital Affiliated to Shandong First Medical University according to Guide for Chinese Ethics Review Committees and informed consent was obtained from all individual participants included in the study. Informed consent Written informed consent was obtained from the patient for publication of their individual details and accompanying images in this manuscript.

\section{References}

1. Abnet CC, Arnold M, Wei WQ (2018) Epidemiology of Esophageal Squamous Cell Carcinoma. Gastroenterology 154(2):360-373

2. Mitin T, Hunter JG, Thomas CR Jr (2015) Esophageal carcinoma. N Engl J Med 372(15):1471-1472

3. Zhu Y et al (2017) Inhibition of HIF-1a by PX-478 suppresses tumor growth of esophageal squamous cell cancer in vitro and in vivo. Am J Cancer Res 7(5):1198-1212

4. Shao JB et al (2019) Hypoxia-inducible factor $1 a$ in combination with vascular endothelial growth factor could predict the prognosis of postoperative patients with oesophageal squamous cell cancer. Pol J Pathol 70(2):84-90

5. Zhang Q et al (2017) Hypoxia-inducible factor-2a promotes tumor progression and has crosstalk with Wnt/ $\beta$-catenin signaling in pancreatic cancer. Mol Cancer 16(1):119

6. Siebel C, Lendahl U (2017) Notch Signaling in Development, Tissue Homeostasis, and Disease. Physiol Rev 97(4):1235-1294

7. Majumder S et al (2021) Targeting Notch in oncology: the path forward. Nat Rev Drug Discov 20(2):125-144

8. Kar R et al., A "NOTCH" Deeper into the Epithelial-To-Mesenchymal Transition (EMT) Program in Breast Cancer. Genes (Basel), 2019. 10(12) 
9. Natsuizaka $\mathrm{M}$ et al (2017) Interplay between Notch1 and Notch3 promotes EMT and tumor initiation in squamous cell carcinoma. Nat Commun 8(1):1758

10. Balamurugan K (2016) HIF-1 at the crossroads of hypoxia, inflammation, and cancer. Int J Cancer 138(5):1058-1066

11. Dang CV et al (2008) The interplay between MYC and HIF in cancer. Nat Rev Cancer 8(1):51-56

12. Albadari N, Deng S, Li W (2019) The transcriptional factors HIF-1 and HIF-2 and their novel inhibitors in cancer therapy. Expert Opin Drug Discov 14(7):667-682

13. Gordon WR et al (2007) Structural basis for autoinhibition of Notch. Nat Struct Mol Biol 14(4):295300

14. Li Y et al (2013) Regulation of EMT by Notch signaling pathway in tumor progression. Curr Cancer Drug Targets 13(9):957-962

15. Jiang $\mathrm{N}$ et al (2020) HIF-1囚-regulated miR-1275 maintains stem cell-like phenotypes and promotes the progression of LUAD by simultaneously activating Wnt/ $\beta$-catenin and Notch signaling.

Theranostics 10(6):2553-2570

16. Yan Y et al (2018) HIF-2a promotes conversion to a stem cell phenotype and induces chemoresistance in breast cancer cells by activating Wnt and Notch pathways. J Exp Clin Cancer Res 37(1):256

\section{Figures}



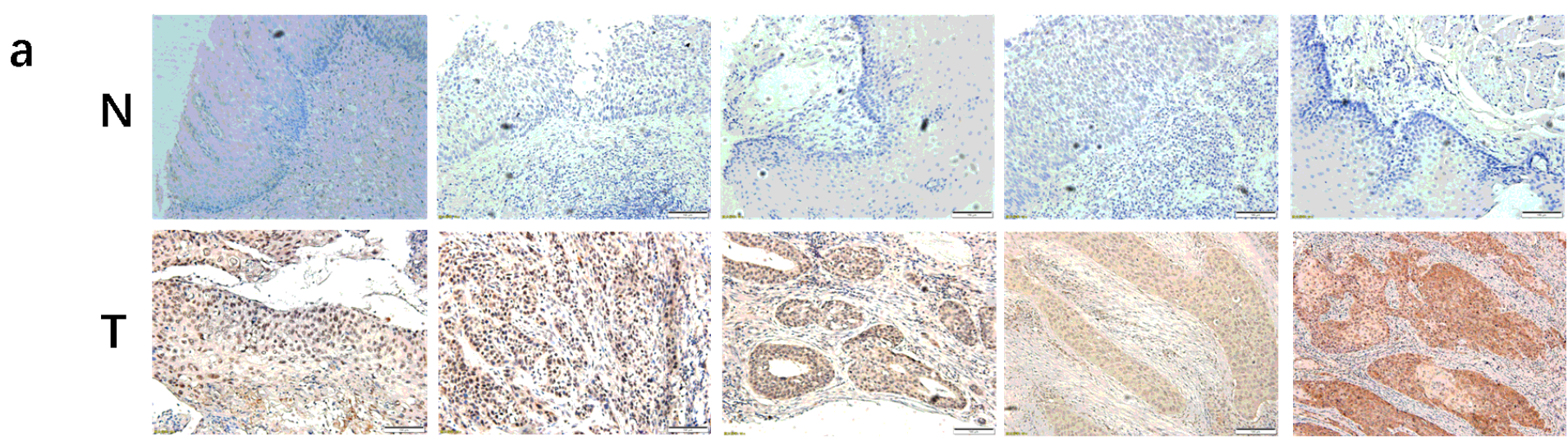

b

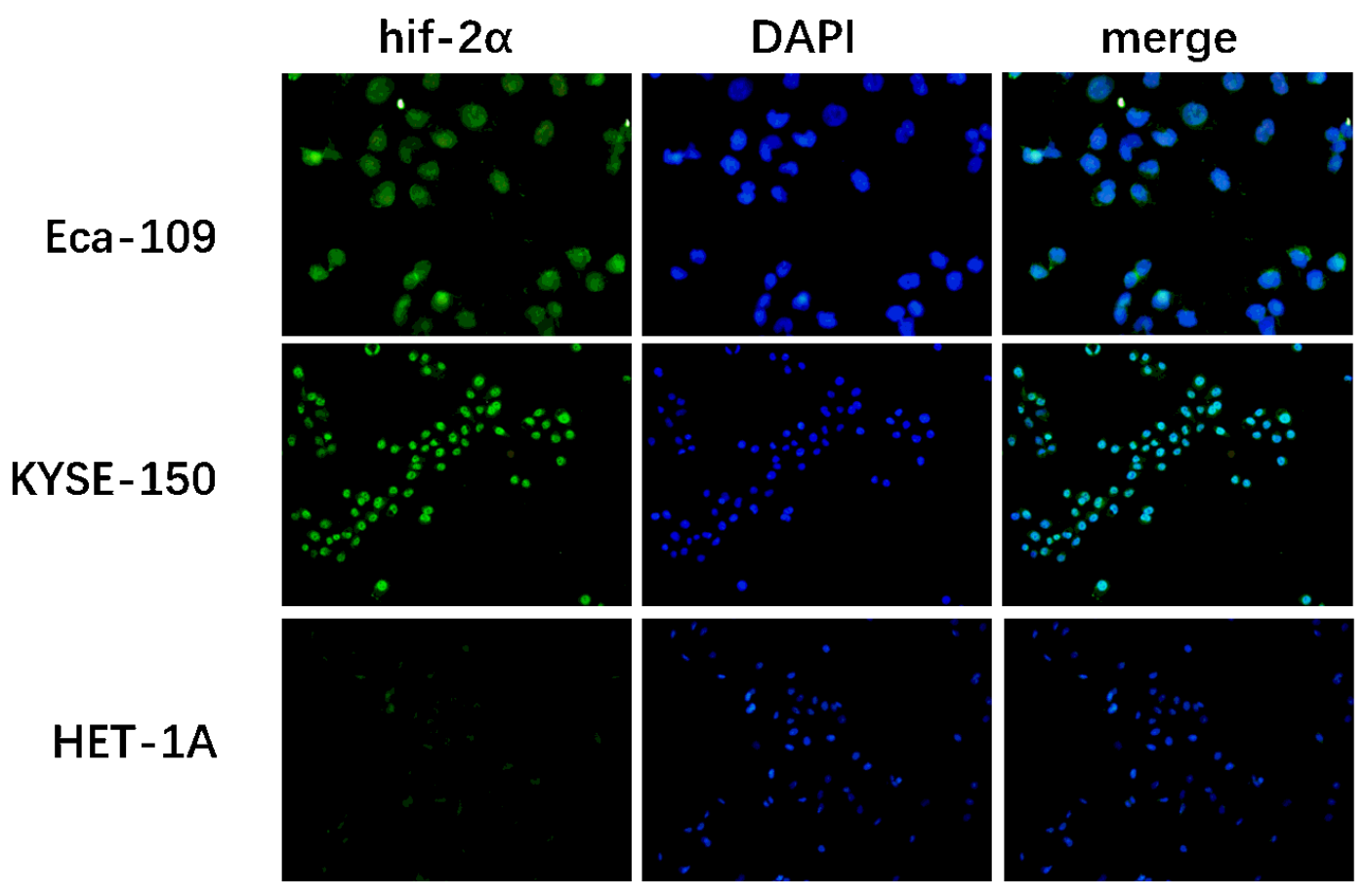

\section{Figure 1}

Hif-2a is overexpressed in ESCC. a. Immunohistochemistry showed the hif-2a level in ESCC tissue is significantly higher than normal tissue. b. Immunofluorescence showed hif-2a was overexpressed in ESCC cells than normal esophageal epithelial cell.

\section{Figure 2}

Hif-2a is associated with hypoxia-induced EMT in ESCC a. morphology changes of ESCC cells after hypoxia treatment for $48 \mathrm{~h}$. b. expression changes of hif-2a and EMT markers in ESCC cells after hypoxia treatment. c. $\mathrm{CoCl} 2$ was used to mimic hypoxia and induce EMT.

\section{Figure 3}


Hif-2a promote proliferation and invasion of esophageal cells. a. CCK8 assay showed silencing hif-2a could inhibit the proliferation of ESCC cells. b. Transwell assay showed silencing hif-2a could reduce the invasion ability of ESCC cells. c. Transwell assay showed overexpressing hif-2a could enhance the invasion ability of ESCC cells. $d$. The cell numbers on the lower surface of the membrane were counted in three randomly selected fields. e. The cell numbers on the lower surface of the membrane were counted in three randomly selected fields.

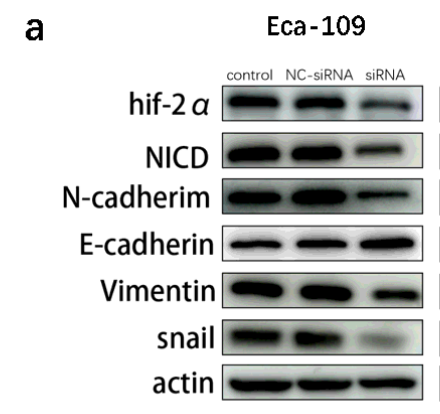

C

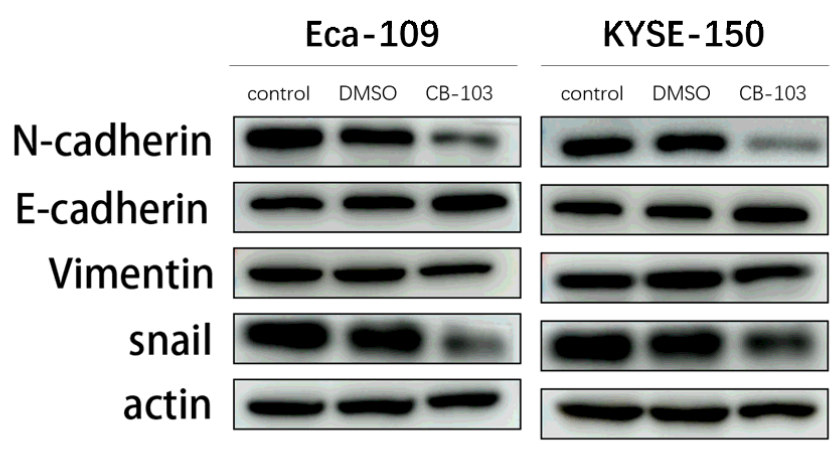

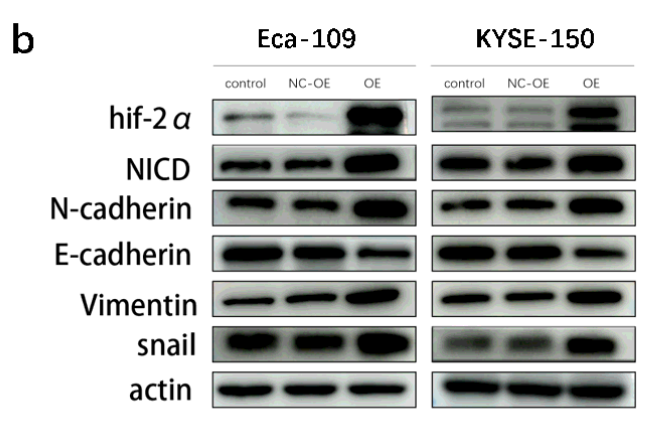

d

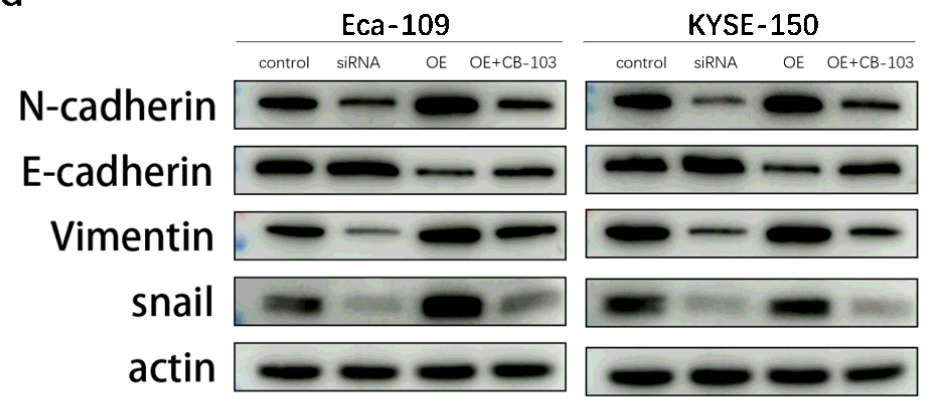

Figure 4

Hif-2a regulate EMT through NOTCH pathway a. Silencing hif-2a reduced Notch signaling and EMT in ESCC cells. b. Overexpressing hif-2a enhanced Notch signaling and EMT in ESCC cells. c. Notch inhibitor reduced EMT in ESCC cells d. Using Notch inhibitor in hif-2a overexpressed cells could reverse the EMT process.

\section{Figure 5}

Silencing hif-2a could suppress the tumor growth in mouse model. a. 1×106 NC-siRNA or sihif-2a Eca-109 cells were injected subcutaneously in the flank of mice. Xenografts from sihif-2a were much smaller compared with NC-siRNA. b. Xenografts from sihif-2a were much smaller compared with NC-siRNA. c. Tumor volume curve of NC-siRNA and sihif-2a. d. Tumor weight of NC-siRNA and sihif-2a. e. The weight of mice injected with NC-siRNA or sihif-2a transfected cells. f. Immunohistochemistry showed expression changes of hif-2a and EMT markers in NC-siRNA or sihif-2a. g. Western blot showed expression changes of hif-2a,NICD and EMT markers in NC-siRNA or sihif-2a. 


\section{Figure 6}

Kaplan-meier curve showed patients with lower expression of hif-2a had significantly longer survival time compared to those with higher expression of hif-2a. 